\title{
Oxysterol signalling is retained in ER-negative and supressed in ER-positive breast cancer
}

\section{Abstract}

Breast cancer treatment and prognosis is informed by biomarker expression. Expression of Oestrogen Receptor-alpha (ER $\alpha)$ for example influences whether the patient receives endocrine- or chemo-therapy. Nutritional status is a modifier of disease free survival and elevated circulating cholesterol associates with increased risk of relapse. Cholesterol hydroxylation produces 'oxysterols' which are selective Liver X Receptor alpha (LXR $\alpha$ ) modulators and ER $\alpha$ agonists. In ER-positive breast cancer, oxysterols induce proliferation and resistance to endocrine therapy, whilst in ER-negative disease oxysterols are anti-proliferative and pro-metastatic suggesting that there are breast cancer subtype specific differences in the genomic targets of the oxysterol-LXR pathway. This study explored the regulation of LXR $\alpha$ signalling in ER-positive and ER-negative breast cancer, and how ligand, receptor and co-factors combine to regulate LXR $\alpha$ target gene expression in different breast cancer types.

In vitro, MDA.MB.468 (ER-negative) cells were more responsive than MCF-7 (ER-positive) cells to synthetic LXR $\alpha$ agonists (T0901317, GW3965) and six oxysterols (22-hydroxycholesterol [22-OHC], 24-OHC, 25-OHC, 27-OHC, 7-ketocholesterol and 24,25-epoxycholesterol), as measured by MTT, LXR-luciferase reporter, and qPCR of canonical targets ABCA1 and APOE (Students t-tests: $\mathrm{p}<0.01$ ). Responses to the antagonist GSK2033 was comparable across cell lines. In vivo, LXRa expression correlated with 48/146 target genes in ER-negative $(n=81)$, but with just 9/146 in ER-positive tumours $(n=234)$ (Fischer exact test: $p<$ 0.0001 ) indicating greater LXRa-mediated transcription of target genes in the aggressive subtype. This was not explained by ligand concentration, as we developed a novel fast oxysterol detection system and found no difference in concentration of 22-OHC, 24-OHC, 25-OHC or 27-OHC between ER-negative $(n=11)$ and ER-positive $(n=11)$ primary tumours obtained from the Leeds Breast Tissue Bank. However, we did observe that expression of LXRa and 2/7 of its co-activators (SRC, TRRAP) were higher in ER-negative relative to ER-positive disease (using TCGA data from cBioPortal) (Mann-Whitney $U$ test: $p<0.001$ ), and that expression of all LXRa co-repressors were lowest in ER-negative disease (NCOR1, NCOR2, LCOR: Mann-Whitney U test: $p<0.001$ for all). siRNA knock-down of NCOR1 and NCOR2 resulted in MCF-7 cells that mimicked the response of MDA.MB.468 cells to oxysterols (as measured by LXR-luciferase and qPCR assay).

These data indicate that despite the anti-proliferative actions of oxysterol-LXRa signalling, there is a, yet to be identified, selective advantage for retention and enhancement of this pathway in ER-negative breast cancer. Dietary routes to selective LXRa modulation (such as plant sterols) may provide patient-led routes to improving ER-negative survival rates.

\section{Conflict of Interest}

There is no conflict of interest 